\title{
Hallazgos característicos por resonancia magnética en síndrome de Sturge Weber y consideraciones cognitivo-conductuales
}

\section{Reporte de un caso}

\author{
Sotomayor Rivas Juan Pablo*, Tapia-Reyna María de Lourdes**
}

\footnotetext{
Resumen

- Presentamos un caso de síndrome de Sturge-Weber en un

- niño de 9 años con cuadros convulsivos, nevus rojo en rostro

- y alteraciones conductuales. Estudios de neuroimagen confir-

- maron el diagnóstico con hallazgos característicos en reso-

- nancia magnética de esta enfermedad. Posteriores pruebas

- psicométricas practicadas al menor demostraron presencia de

- capacidad mental deficiente para la edad, así como retraso

- madurativo. La resonancia magnética, con sus técnicas avanza-

- das, es el método de imagen ideal para la valoración de estos

- pacientes. Además, en casos como el presente, la valoración del

- área cognitiva permite determinar su estado mental y ayuda

- al clínico a decidir sobre la utilidad y las implicaciones de un

- tratamiento quirúrgico posterior. LUX MÉDICA AÑO 9, NÚMERO 28,

SEPTIEMBRE-DICIEMBRE 2014, PP 33-38.
}

Palabras clave: Síndrome Sturge-Weber, facomatosis, Resonancia Magnética.

\section{Abstract}

We present a case of Sturge-Weber syndrome in a 9-year child with seizure disorders, red face nevus and behavioral disturbances. Neuroimaging studies confirmed the diagnosis with characteristic findings of this disease. Subsequent psychometric tests confirmed the presence of lower mental capacity to age and developmental delay. Magnetic resonance imaging, with its advanced techniques, is the ideal method for the assessment of these patients. Moreover, in cases like this, the assessment of cognitive functions determines his mental state and helps the clinician to decide on the usefulness and implications of further surgical treatment. LUX MÉDICA AÑO 9, NÚMERO 28, SEPTIEMBREDICIEMBRE 2014, PP 33-38.

\section{Introducción}

En 1860 el doctor Rudolf Schirmer describió el caso de un paciente con un extenso nevo facial que involucraba ambos lados del rostro, se relacionaba con hidroftalmos izquierdo y dilataciones varicosas de las venas retinianas. Posteriormente, en 1879, el doctor William Allen Sturge describió el caso de una niña con nevo rojo en el lado

Médico radiólogo, coordinador del Departamento de Tomografia y Resonancia Magnética en Diagnóstico Médica Integral, Siglo XXI, Aguascalientes, Ags. México

** Estudiante del sexto semestre de la carrera de psicología de la Universidad La Concordia.

Fecha de recibido: 8 abril 2014

Fecha de aceptación: 23 octubre 2014

Correspondencia Dr Juan Pablo Sotomayor Rivas. Emiliano Zapata No 532 Zona Centro CP 20000 Aguascalientes, Ags., México. Teléfono (449)9102930 extensión 165 Correo electrónico drsotomayor@siglo21.mx 
derecho de la cabeza y la cara que se asociaba a dilatación del globo ocular derecho y cambios vasculares de la coroides. Además la paciente sufría de epilepsia parcial del hemicuerpo izquierdo, lo que el doctor Sturge atribuyó a una probable lesión vascular asociada en el hemisferio cerebral derecho. ${ }^{1,2}$

El primero en publicar los hallazgos radiológicos típicos de un paciente con alteraciones faciales similares, en placa simple de cráneo, fue Frederik Parkes Weber en 1922, quien describió la presencia de calcificaciones curvilíneas que seguían el patrón de los surcos corticales del encéfalo. ${ }^{1,2}$

Vicente Dimitri, radiólogo argentino, también aportó datos sobre las características radiográficas de la enfermedad en el año de $1923 .{ }^{1}$

En la actualidad, el padecimiento se conoce comúnmente como síndrome de Sturge-Weber. Sin embargo, el término debe de utilizarse únicamente en los casos que presentan dos o más signos mayores tales como nevo facial (a veces descrito como mancha en vino de Oporto), angioma intracraneal y angioma coroideo ocular. El nevo suele distribuirse en el territorio de las ramas trigeminales. ${ }^{2-4}$

Existen múltiples sinónimos (por ejemplo angiomatosis encéfalo-trigeminal, angiomatosis meníngea capilar o enfermedad de Dimitri). Sin embargo, posiblemente la forma más correcta de referirse a esta combinación de alteraciones, dejando de lado los epónimos, sea angiomatosis meníngea oculo-facial o angiomatosis meningofacial. ${ }^{3}$

Por otra parte, al considerar que la patología involucra tanto la piel como los ojos y el sistema nervioso central, se le cuenta entre el grupo de padecimientos conocidos como facomatosis, o síndromes neuro-cutáneos. En esta categoría se encuentran enfermedades tales como la esclerosis tuberosa (enfermedad de Bourneville), la enfermedad de Von Hippel-Lindau y la ataxia-telangiectasia, entre otras. ${ }^{3,4}$

La etiología del Sturge-Weber es desconocida. Se cree que las malformaciones de los capilares y capilares venosos tienen su origen en mutaciones somáticas del neuroectodermo en el periodo fetal. Suele aparecer de manera esporádica aunque se han descrito casos heredados de manera autosómica recesiva y dominante. El angioma facial suele ser unilateral, pero puede ser bilateral del $14 \%$ al $30 \%$ de los casos. El angioma ocular sucede en el $30 \%$ de los casos. Las crisis convulsivas afectan del 71 al $90 \%$ de los pacientes. El retraso mental se encuentra presente entre el 50 y el $70 \%$ de los casos. ${ }^{2,3}$

Clínicamente, los pacientes presentan además glaucoma aproximadamente en un $70 \%$ de los casos en el globo ocular del lado afectado e incluso hemiparesia y hemiatrofia corporal. ${ }^{3}$

La presencia de angioma leptomeníngeo en la piamadre se relaciona con alteración neurológica ya que existe atrofia cerebral 


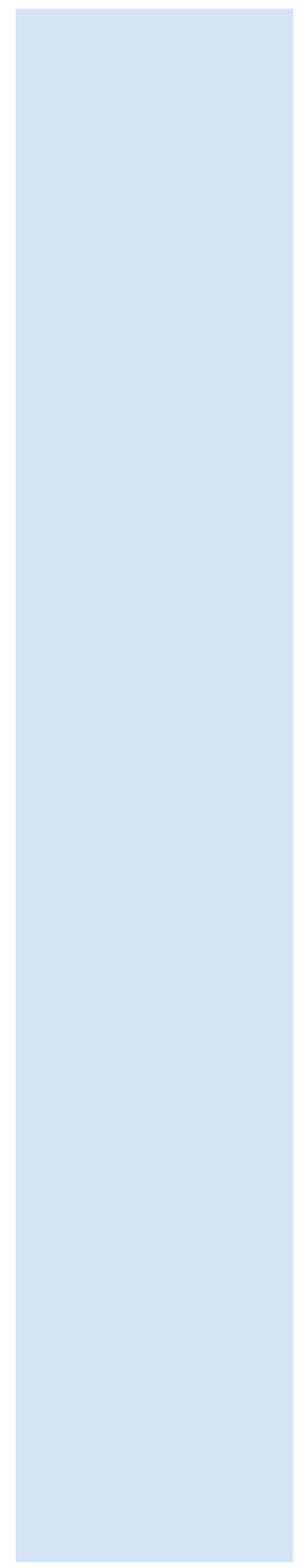

debido a gliosis por isquemia, y el angioma disminuye el recambio sanguíneo del área. ${ }^{3}$

Los estudios de imagen como placa simple y tomografía computada (TC) muestran calcificaciones corticales giriformes cercanas a los angiomas, las cuales afectan con mayor frecuencia los lóbulos occipitales, no obstante, también es posible que se vean afectados lóbulos temporales y parietales. La alteración de los lóbulos frontales es rara. Las calcificaciones son secundarias a isquemia tisular. Generalmente la angiomatosis intracraneal es unilateral, pero puede ser bilateral en algunos casos. La TC permite identificar las calcificaciones, así como la atrofia cerebral cuando ésta ya es un tanto avanzada. La resonancia magnética (RM) es capaz de identificar la atrofia cerebral en etapas más tempranas con alteración en intensidad de señal de la sustancia blanca en secuencias de líquido claro (como T2 y Flair), además de que las fases con efecto angiográfico venoso y arteria suelen identificar la presencia de angiomatosis, la ausencia de venas corticales superficiales y los vasos colaterales prominentes que involucran al sistema venoso profundo. Las secuencias de eco gradiente y T2 muestran zonas hipointensas a nivel de las calcificaciones corticales. La aplicación de medio de contraste igualmente pone de manifiesto la presencia de angiomatosis leptomeníngea. Estas características vuelven a la RM el método de imagen ideal para la valoración estructural del sistema nervioso central en los pacientes con esta enfermedad. Otros estudios paraclínicos, como tomografía con emisión de positrones (PET), tomografía con emisión de fotón simple (SPECT) y el electroencefalograma, aportan datos adicionales funcionales y sobre el metabolismo cerebral en estos casos. ${ }^{1-4}$

Para el tratamiento se toma en cuenta la edad del paciente al momento del diagnóstico así como el grado de alteraciones neurológicas presentes. Se puede ofrecer tratamiento médico conservador con el objetivo primordial de controlar las crisis convulsivas, o en otras ocasiones, cuando las crisis son incoercibles, se considera la realización de resección quirúrgica, lobectomía o incluso hemisferectomía. ${ }^{1,3}$

\section{Presentación del caso clínico}

Paciente masculino de 9 años de edad. Acude de urgencia a valoración por haber sufrido caída de su propia altura, con traumatismo craneal y pérdida del estado de alerta hace cinco horas aproximadamente. Al momento se encuentra consciente, activo y reactivo, sin déficits neurológicos, muestra escoriaciones epidérmicas leves. Llama la atención la presencia de nevus color rojo, plano, en el lado derecho de la cara, sin hipertrofia de partes blandas. Se interroga a la madre que refiere como antecedentes que el niño es distraído en la escuela, con bajas calificaciones y cierto 
comportamiento agresivo hacia sus compañeros, lo cual atribuyen al carácter propio del menor. Ha sufrido en toda su vida cinco episodios de cuadros convulsivos a decir de la mamá, según su descripción de tipo tónicoclónicas, parciales simples. Médico general de su localidad atribuyó las crisis a probable neurocisticercosis, por lo que le prescribió en una ocasión albendazol. Actualmente no recibe tratamiento farmacológico alguno. Como dato adicional se destaca que nadie presenció la caída del menor. Sus hermanos lo encontraron inconsciente en el piso afuera de su casa. El niño vive en medio rural, es el menor de seis hermanos, obtenido por parto eutócico atendido en casa por partera, con cuadros completos de esquema de vacunación. No hay antecedentes heredofamiliares de importancia.

\section{Hallazgos radiológicos}

Las imágenes de TC mostraron la presencia de cuatro imágenes hiperdensas irregulares, gruesas, en la base del lóbulo temporal derecho de $17 \times 12,5 \times 6,4 \times 5$ y de $8 \times 5 \mathrm{~mm}$, con densidad de $300 \mathrm{HU}$. Se identifican dos zonas hiperdensas de $5 \mathrm{~mm}$ en el lóbulo frontal derecho, poco definidas, las cuales no se puede precisar si corresponden a calcificaciones, a hematomas o zonas de contusión hemorrágica. No hay evidencia de hematomas epi o subdurales ni se observa hemorragia subaracnoidea.

Debido a los hallazgos no concluyentes, se efectuó posteriomente estudio de resonancia magnética donde se observó aumento de profundidad de surcos en torno a los lóbulos temporal y parietal derechos en relación con pérdida de volumen cortical por atrofia focal y pequeñas áreas de isquemia en sustancia blanca (Imagen 1). Tras la aplicación de contraste se observó un intenso realce leptomeningeo por angiomatosis, que sigue el contorno de los giros corticales a nivel temporo-parietal en el hemisferio derecho (Imágenes 2, 3 y 4). Se aprecian venas subependimarias prominentes, las cuales proporcionan drenaje colateral venoso que dirigen al seno recto en secuencia angiográfica venosa (Imagen 5 ). En estudio de eco gradiente se observaron imágenes hipointensas correspondientes a calcificaciones en lóbulo frontal derecho y en la base del lóbulo temporal ipsilateral, sin características de hemorragia o hematoma en otras secuencias. Se observa que los plexos coroideos son asimétricos, con mayores diámetros del plexo en el ventrículo lateral derecho. Los presentes hallazgos asociados a las calcificaciones gruesas observadas en estudio de TAC son compatibles con síndrome de Sturge-Weber.

\section{Comentario psicológico}

Como parte de la evaluación integral del paciente, se le practicaron pruebas psicométricas así como de maduración infantil. Para tal efecto se eligió en primer lugar el test de matrices progresivas coloreadas de Raven, considerando principalmente su aspecto no cultural (ya que el paciente proviene de un entorno principalmente rural), así como lo atractivo que resulta el material para los niños y el tiempo relativamente corto que se requiere para su aplicación. Posteriormente se aplicó en otra cita el test de Bender para el desarrollo mental, igualmente teniendo en cuenta la sencillez de su diseño y tiempo de aplicación. 5,6

El niño obtuvo un resultado bajo de 21 puntos en la prueba de Raven con diagnóstico de capacidad mental deficiente.

En el test de Bender se obtuvo un desarrollo madurativo anormal, con retraso aproximado de 3 años inferior a la edad cronológica. 
Estos resultados permitieron establecer, en conjunto con las distintas pruebas médicas, la presencia de daño orgánico cerebral y así explicar el mal desempeño escolar y las alteraciones conductuales del paciente que erróneamente se habían interpretado como parte de un "carácter difícil".

Este fue un paso importante para el completo diagnóstico del paciente, primeramente porque permite diseñar estrate- gias psicopedagógicas para mejorar tanto el desempeño académico como el entorno familiar y afectivo del niño; además de que el nivel de inteligencia fluida y grado de madurez mental son factores considerados por los neurocirujanos para decidir si el paciente es candidato a procedimientos quirúrgicos. Y de ser así, también sirven como puntos de referencia del estado previo y posquirúrgico del paciente.

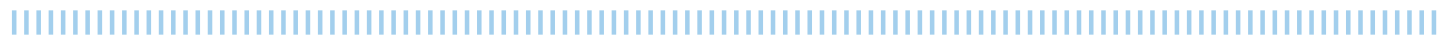

\section{Discusión}

El paciente de nuestro caso presentaba datos clínicos y signos característicos del síndrome de Sturge-Weber, sin embargo el diagnóstico se estableció de manera tardía. Debemos de recordar que es importante establecer el diagnóstico lo más pronto posible a fin de ofrecer al paciente el mejor de los tratamientos posibles y mejorar su calidad de vida. Las imágenes de RM facilitan la comprensión de la serie de cambios y eventos que se suceden en el encéfalo ante la presencia de angiomatosis meníngea incluyendo atrofia cerebral, el desarrollo de calcificaciones distróficas, la disminución de venas corticales cerebrales ipsilaterales a la angiomatosis y la presencia, o no, de drenaje anómalo al sistema venoso profundo.
Debido a la complejidad de las alteraciones presentes en estos casos, que involucran al área cognitiva y la conducta, se recomienda trabajar en conjunto con psicología. Así, el psicólogo determinará el status mental del paciente. Esto es de suma importancia, ya que los pacientes que muestran retraso mental profundo no son candidatos a tratamiento quirúrgico por lo general.

Nuestro paciente sufría de capacidad mental deficiente además de retraso en el desarrollo madurativo. Sin embargo no presentaba cuadros convulsivos frecuentes ni intensos por lo que se optó por un tratamiento médico conservador inicial y vigilancia.

\section{Conclusiones}

El correcto diagnóstico de los pacientes con síndrome de Sturge-Weber exige tanto una adecuada exploración clínica oportuna como la evaluación estructural del sistema nervioso central mediante estudios de imagen como la RM, pruebas funcionales y una evaluación psicológica detallada y precisa. De la correcta conjunción de estos factores depende la elección del tratamiento óptimo del paciente. La RM es sus distintas modalidades es el método de imagen adecuado en la evaluación estructural del sistema nervioso central en pacientes con síndrome de Sturge-Weber. 


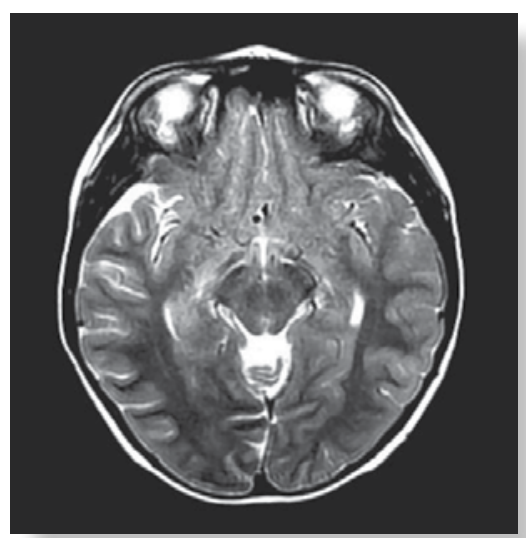

Imagen 1. Imagen de RM secuencia T2, planos axial. Se observa aumento en la profundidad de los surcos corticales en el lóbulo temporal derecho que indica pérdida de volumen cortical y atrofia.

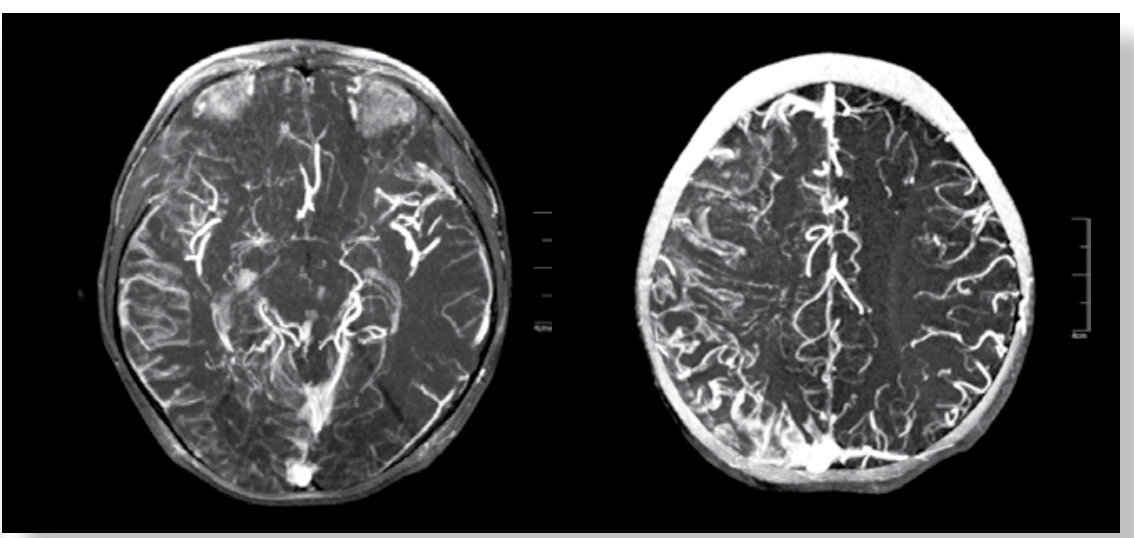

Imágenes 2 y 3 . Imágenes de RM en secuencias $3 D$ TOF con efecto angiográfico arterial en plano axial que muestran intenso realce meníngeo con angiomatosis leptomeníngea.

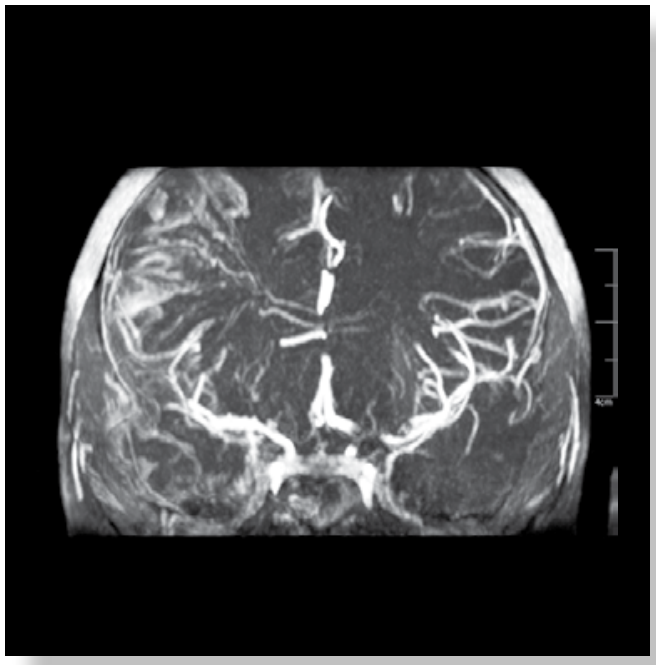

Imagen 4. Imagen de RM 3D TOF con efecto angiográfico arterial plano coronal. Asimetría de estructuras vasculares por angiomatosis leptomeníngea.

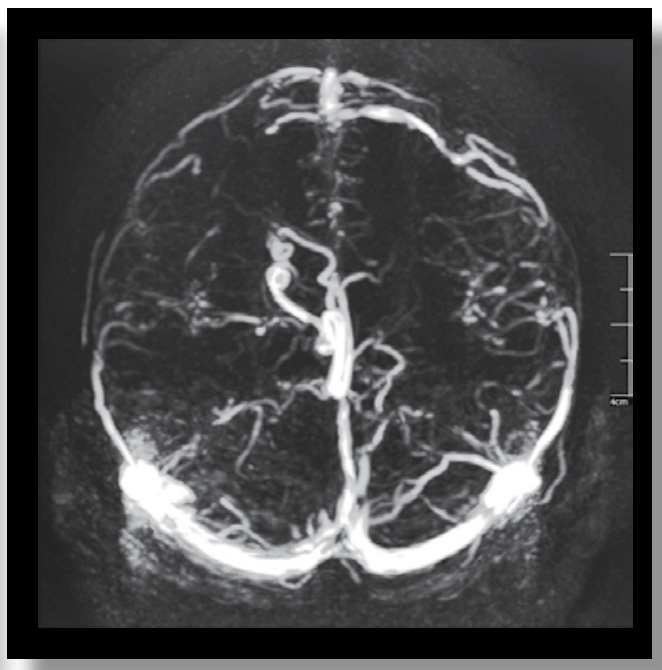

Imagen 5. Imagen de RM 3D TOF con efecto angiográfico venoso. Se identifican venas subependimarias prominentes en hemisferio cerebral derecho que se dirigen al seno recto, proporcionando drenaje venoso colateral a la angiomatosis leptomeníngea. Nótese la falta de venas superficiales corticales sobre el hemisferio derecho.

\section{Bibliografía}

1.- Falconer MA, Rushworth RG. Treatment of encephalotrigeminal angiomatosis (Sturge-Weber disease) by hemispherectomy. Guy's Maudsley Neurosurgical Unit, London 1959. On-line in adc.bmj.com on November 23, 2013

2.- Miranda $M$, Güemes $H$, Barbero $A$, et al. Síndrome de Sturge-Weber: Experiencia en 14 casos. Anales españoles de pediatría 1997; 46 (2): 138-142.

3.- Fernandez C, Gómez G, Sardiñaz H. Síndrome de Sturge-Weber. Revisión. Rev Cubana Pediatría 1999; 71 (3):153-159.
4.- Martínez G, López L, Pérez B. Síndrome de SturgeWeber: Combinación de lesiones angiomatosas coroideas y orbitarias en un paciente. Arch Soc Esp Oftalmol 2008; 83: 249-232.

5.- Ramirez B, Lorenzo G, Díaz B. Matrices progresivas de Raven y su versión abreviada en la población escolar. Revista Mexicana de Neurociencias 2013; 14 (2):63-67.

6.- Heredia A. Santaella H. Somarriba R. Interpretación del test gestáltico visomotor de Bender sistema de puntuación de Koppitz. 2012. Textos de apoyo didáctico psicodiagnóstico I, quinto semestre, Facultad de psicología UNAM. México. 